\title{
Öffentlich-rechtliche Lebensversicherungsanstalten
}

Verband öffentlicher Lebensversicherungsanstalten

in Deutschland

Verbandsvorsitzender:

Dr. Karl Wilder, Generaldirektor der Anstalt Schleswig-Holstein

Verbandsvorstand:

Dr. Herbert Dibbert (o), Dr. Alfred John (o)

Verbandsmathematiker: Dr. Herbert Dibbert

1. Hessen-Nassau

Vorstand: Dr. Dr. Oskar Schulz (V), Dr. Gerhard Knüfermann (o). Chefmathematiker: Dr. Gerhard Knüfermann.

2. Rheinprovinz

Vorstand: Dr. Anton Bertgen (V), Dr. Wilhelm Kampmann (o). Chefmathematiker: Walter Kehr.

3. Westfalen

Vorstand: Dr. Heinz Herberholz (V), Kurt Hellwig (o), Dr. Helmut Becker (0).

Anstaltsmathematiker: Eberhard Voigt.

4. Schleswig-Holstein

Vorstand: Dr. Karl Wilder (V), Bruno Galenschewsky (st).

Mathematiker: Gerhard Köhler.

5. Hannover

Vorstand: Dr. Hans Thunsdorff (V), Dr. Franz Härdrich (o), Hermann Drößler (st), Dr. Erich Kreuter (st).

6. Bayern

Vorstand: Dr. Rudolf Zorn (V), Dr. Michael Daubenmerkl (o), Hans Lochner (o), Dr. Josef Velhorn (st).

Chefmathematiker: Dr. Rudolf Bernstetter.

7. Oldenburg

Vorstand: Dr. Erich Muckelmann (V), Friedrich Humbert (o).

Chefmathematiker: Friedrich Humbert.

8. Baden

Vorstand: Alfred Tröbliger (V), Dr. jur. Hans Schleenbecker (0). Mathematiker: Dr. Werner Müller.

9. Braunschweig

Vorstand: Dr. Hans Goetz (o), Dr. Ernst Riesel (o), Reinhold Jausel (st).

Chefmathematiker: Reinhold Jausel.

10. Sparkassenversicherung

Vorstand: Gustav Ernst (V), Erwin Fix (o).

Mathematiker: Rolf Hoffmann

11. Berlin

Vorstand: Dr. Willy Muller-Wieland (V), Gerhard Loch (st).

Chefmathematiker: Erich Friede. 